\title{
Respostas do treinamento sensório-motor no equilíbrio e coordenação de crianças com deficiência visual
}

\section{Answers of training sensory motor in balance and coordination children with visual impairment}

\author{
Arilene Maria da Silva Santos; Roberta Lillyan Rodrigues Reis²; João Alberto Costa Neto3; Tásia \\ Peixoto de Andrade Ferreira; ${ }^{4}$ Marcelino Martins ${ }^{5}$ \\ 1 Residência multiprofissional em saúde da família e comunidade - Universidade Estadual do Piauí - UESPI. Teresina, PI - Brasil. \\ 2 Programa de Pós-graduação em Biotecnologia - Universidade Federal do Piauí - UFPI. Parnaíba, PI - Brasil. \\ 3 Residência multiprofissional em terapia intensiva do adulto. Universidade Estadual do Piauí - UESPI. Teresina, PI - Brasil. \\ 4 Departamento de Fisioterapia. Christus Faculdade do Piauí - CHRISFAPI. Piripiri, PI - Brasil. \\ 5 Centro de Ciências da Saúde, Faculdade de Ciências Médicas - Universidade Estadual do Piauí - UESPI. Teresina, PI - Brasil. \\ Endereço para correspondência: \\ Arilene Maria da Silva Santos. \\ Quadra 06 Casa 15 Bairro Bela Vista I \\ 64030-020 - Teresina - PI [Brasil] \\ arilenemaria@hotmail.com
}

\begin{abstract}
Resumo
Introdução: A presença de restrições visuais na infância leva a desvios e dificuldades nas habilidades sensório-motoras. Objetivos: Verificar as respostas do treinamento sensório-motor no equilíbrio e coordenação de crianças com deficiência visual. Métodos: Trata-se de um relato de casos não controlado, transversal. A amostra foi composta por duas crianças em idade escolar, com diagnóstico oftalmológico de baixa visão e cegueira total. Foram aplicados o Teste de equilíbrio de Berg e o Teste de Coordenação Corporal para Crianças - Bateria de Testes KTK antes e após 10 atendimentos com duração média de 50 minutos, duas vezes na semana. Resultados: Para a Criança 1 observou-se melhora de aproximadamente $11 \%$ para coordenação e $13 \%$ no equilíbrio; e para a Criança 2 verificou-se aumento de $17 \%$ na coordenação, e $6 \%$ para o equilíbrio. Conclusão: O protocolo utilizado trouxe benefícios para o equilíbrio e resposta motora destas crianças com deficiência visual.
\end{abstract}

Descritores: Criança; Pessoas com deficiência visual; Desempenho psicomotor; Equilíbrio postural; Fisioterapia.

\begin{abstract}
Introduction: The presence of visual restrictions in childhood leads to deviations and difficulties in sensorimotor skills. Objectives: To verify the responses of sensorimotor training in the balance and coordination of children with visual impairment. Methods: This is an uncontrolled, transverse cases report. The sample consisted of two school-age children, with an ophthalmologic diagnosis of low vision and total blindness. The Berg Balance Test and the Body Coordination Test for Children - KTK Test Battery were applied before and after 10 visits, average duration of 50 minutes, twice a week. Results: Child 1 showed improvement of approximately $11 \%$ for coordination and $13 \%$ for balance; and for Child 2 there was an increase of $17 \%$ in coordination, and $6 \%$ for balance. Conclusion: The protocol used brought benefits to the balance and motor response of these visually impaired children.
\end{abstract}

Keywords: Child; Visually impaired persons; Psychomotor performance; Postural balance; Physical therapy specialty. 


\section{Introdução}

O sentido da visão exerce um importante papel na estabilização do equilíbrio corporal e coordenação motora, por fornecer ao sistema nervoso informações sobre a direção e velocidade dos movimentos e posição dos segmentos do corpo em relação a eles mesmos e ao ambiente. Tais considerações indicam a importância da visão na capacidade motora, tornando-a mais difícil quando há alguma deficiência visual, principalmente na infância ${ }^{1,2,3}$.

A presença de restrições visuais na infância leva a desvios e dificuldades nas habilidades sensório-motoras, prejudicando assim o controle corporal e capacidade motora ${ }^{4}$. As crianças com deficiência visual possuem limitações à capacidade de movimento livre, seguro e confiante no ambiente. Dessa maneira, apresentam déficit de equilíbrio, mobilidade, além de coordenação motora prejudicada ${ }^{5,6}$.

A falta de controle visual para autocorreção leva a um reajuste do sistema nervoso central através de outros sistemas ${ }^{7,8}$ que são suscetíveis a estimulação, sabendo-se da capacidade de adaptação do sistema nervoso por meio de experiências e atividades, espera-se que o equilíbrio corporal e coordenação motora adequados possam ser alcançados a partir de um treinamento relacionando informação sensorial e ação motora, como no treino sensório-motor ${ }^{9,10}$.

Diante da escassez de pesquisas e publicações na área e da relevância associada à comum presença de disfunções sensoriais e motoras em tais crianças, este estudo teve o objetivo de verificar as respostas do treinamento sensóriomotor no equilíbrio e coordenação de crianças com deficiência visual.

\section{Material e métodos}

Trata-se de um relato de casos não controlado, transversal. O estudo foi realizado em uma Associação filantrópica de habilitação e reabilitação de pessoas com deficiência visual no município de Teresina-PI, no período de julho a setembro de 2015. Os critérios de inclusão foram a assinatura do Termo de Consentimento Livre e Esclarecido pelos responsáveis, crianças com diagnóstico de baixa visão ou cegueira que estavam devidamente matriculadas e frequentando regularmente a instituição onde foi realizada a pesquisa, que possuíssem marcha independente e não apresentassem outras doenças associadas e/ou comorbidades. O critério de exclusão utilizado foram as crianças que somaram o total de três faltas consecutivas ao atendimento, sem nenhuma justificativa prévia. A presente pesquisa foi aprovada com o número do parecer 1.034.776 pelo Comitê de Ética em Pesquisa da Universidade Estadual do Piauí (UESPI).

A amostra foi obtida por conveniência, de caráter intencional, sendo incluídas todas as crianças que frequentavam regularmente a instituição no período de realização da pesquisa. Foram incluídas três crianças em idade escolar, as quais apresentavam marcha independente e estavam matriculadas e frequentando regularmente a Associação, sem qualquer outra doença associada. Com perda amostral de uma criança por abandonar os atendimentos, somando o total de três faltas consecutivas sem nenhuma justificativa prévia. Participaram do estudo duas crianças, a Criança 1 (de sete anos) e a Criança 2 (de onze anos de idade), com diagnóstico oftalmológico de baixa visão e cegueira total, respectivamente.

Foram aplicados testes específicos para graduar quantitativamente o equilíbrio e coordenação corporal: Teste de equilíbrio de Berg (escala com 14 itens que vão de 0 a 4 pontos e pontuação total máxima de 56), não sendo considerada, na criança com diagnóstico de cegueira total, a sexta tarefa: de pé com os olhos fechados (portanto pontuação máxima de 52); e o Teste de Coordenação Corporal para Crianças - Bateria de Testes KTK, sendo aplicadas duas tarefas (Trave de Equilíbrio e Saltos Laterais), que não exigem controle visual em sua execução, sendo por isso adequada a sua aplicação para crian- 
ças com deficiência visual ${ }^{11}$. Foi determinado o coeficiente motor final pelo somatório dos referentes à cada tarefa, permitindo classificar o nível de desempenho coordenativo. Com relação a esta classificação por meio do Teste KTK, de acordo com Carminato ${ }^{12}$, o escore final segundo tabela do próprio teste permite classificar como: "perturbações da coordenação" o resultado com escore final menor que 70; como "insuficiência coordenativa" pontuação de 71 a 85; como "coordenação normal" de 86 a 115; de 116 a 130 considera-se como "coordenação boa"; e de 131 a 145 como "coordenação muito boa".

Após avaliação inicial, as crianças foram submetidas ao treinamento sensório motor que compreendeu 10 atendimentos com duração média de 50 minutos, duas vezes na semana. Um protocolo de exercícios (Tabela 1) foi elaborado para os atendimentos. Ao final, realizada nova avaliação para comparação.

Após a coleta de dados e revisão dos dados mensuráveis, estes foram organizados, classificados, agrupados e armazenados em banco construído em uma planilha eletrônica, no programa Microsoft Office Excel.

\section{Resultados}

$\mathrm{Na}$ avaliação inicial para a Criança 1, a pontuação obtida no teste de coordenação corporal foi de 168 (somatório dos coeficientes trave de equilíbrio:106 e saltos laterais: 62), o que é referente, segundo o manual da Bateria de Testes KTK, à pontuação de 75 como escore final, portanto, classificando-a com insuficiência coordenativa. Na avaliação final, ou seja, após os dez atendimentos, o somatório dos coeficientes do KTK aumentou para 191(trave de equilíbrio: 119 e saltos laterais: 72), obtendo-se 83 como escore segundo manual do teste KTK, sendo ainda seu desempenho classificado como insuficiência coordenativa, porém observandose que houve melhora de aproximadamente $11 \%$ na pontuação do referido teste de coordenação motora.

Para a Criança 2, o somatório dos coeficientes do teste de coordenação corporal na avaliação inicial foi de 132 (trave de equilíbrio:77 e saltos laterais:55) resultando em 63 como escore final, esta criança então obtendo pior pontuação e consequente classificação de perturbações da coordenação, também segundo tabela do teste de coordenação aplicado. Em sua reavaliação, após o treinamento sensóriomotor, o somatório dos coeficientes do KTK aumentou para 165 (trave de equilíbrio: $101 \mathrm{e}$ saltos laterais: 64), referente ao escore de 74 , melhorando seu desempenho coordenativo em $17 \%$ e alcançando a classificação de insuficiência coordenativa.

Quanto ao equilíbrio utilizando-se a Escala de Berg (Tabela 2), a Criança 1 atingiu contagem inicial de 45 , obtendo a soma de pontuação 4 em

Tabela 1: Protocolo de exercícios utilizado

\begin{tabular}{c|c|c}
\hline Proposta de exercício & Objetivo & Tempo e repetições \\
\hline Deambular sobre antepé & Treino de equilíbrio & 10 repetições ir e voltar \\
\hline Caminhada sobre solos instáveis com estímulos sensoriais & Treino de equilíbrio rápido e estímulo proprioceptivo & 10 repetições ir e voltar \\
\hline Deslizar pés sobre bolas de propriocepção & Estímulo proprioceptivo & 3 minutos \\
\hline Deambular sobre colchonete & Aprimorar equilíbrio e coordenação & 10 repetições ir e voltar \\
\hline Caminhar sobre aquatubo/ "macarrão" & Estimular coordenação e equilíbrio & 10 repetições ir e voltar \\
\hline Pular corda e pular entre bambolês & Estimular coordenação e equilíbrio & 10 repetições \\
\hline Pular sobre colchonete & Melhorar propriocepção e treino de reequilíbrio & 2 minutos \\
\hline Apoio na tábua de equilíbrio & Propriocepção e equilíbrio & 4 minutos \\
\hline Equilibrar-se sobre bola suíça & Treino de reajuste rápido & 4 minutos \\
\hline
\end{tabular}

Fonte: Adaptado de Sá, Bim7; Lopes, Kitadai e Okai ${ }^{13}$ 
sete tarefas, de 3 em cinco tarefas, 2 em uma das atividades do teste e pontuação 0 para outra. Após o treinamento o valor total se modificou para 51, aumentando para dez a quantidade de itens com pontuação 4, pontuação 3 em três tarefas e obtendo-se 2 em uma tarefa, de forma que não zerou nenhuma atividade.

A Criança 2 alcançou como equilíbrio inicial o total de 46 pontos, marcando 4 em oito itens, 3 para quatro tarefas e 2 pontos em uma das tarefas. Na avaliação final aumentou sua pontuação total para 49, obtendo 4 em dez tarefas e 3 nas demais.

Tabela 2: Pontuação do teste de equilíbrio de Berg pré e pós treinamento

\begin{tabular}{|c|c|c|c|c|}
\hline \multirow{2}{*}{ Atividade } & \multicolumn{2}{|c|}{ Criança 1} & \multicolumn{2}{|c|}{ Criança 2} \\
\hline & Pré & Pós & Pré & Pós \\
\hline 1. Sentado para em pé & 4 & 4 & 4 & 4 \\
\hline 2. Em pé sem apoio & 4 & 4 & 4 & 4 \\
\hline 3 Sentado sem suporte para as costas & 4 & 4 & 4 & 4 \\
\hline 4. Em pé para sentado & 3 & 3 & 3 & 3 \\
\hline 5. Transferências & 3 & 4 & 3 & 3 \\
\hline $\begin{array}{l}\text { 6. Em pé sem suporte com olhos } \\
\text { fechados }\end{array}$ & 4 & 4 & - & - \\
\hline 7. Em pé sem suporte com pés juntos & 4 & 4 & 4 & 4 \\
\hline $\begin{array}{l}\text { 8. Alcance a frente com braços } \\
\text { estendidos }\end{array}$ & 2 & 3 & 4 & 4 \\
\hline 9. De pé apanhar objeto do chão & 4 & 4 & 4 & 4 \\
\hline 10. Em pé virar-se sobre os ombros & 3 & 4 & 3 & 4 \\
\hline 11. Virar $360^{\circ}$ & 4 & 4 & 4 & 4 \\
\hline $\begin{array}{l}\text { 12. Colocar pés alternados sobre } \\
\text { degrau }\end{array}$ & 3 & 4 & 3 & 4 \\
\hline $\begin{array}{l}\text { 13. Permanecer de pé sem apoio com } \\
\text { pé a frente }\end{array}$ & 0 & 2 & 4 & 4 \\
\hline $\begin{array}{l}\text { 14. Permanecer em pé apoiado em } \\
\text { uma perna }\end{array}$ & 3 & 3 & 2 & 3 \\
\hline Total & 45 & 51 & 46 & 49 \\
\hline
\end{tabular}

Fonte: Elaborada pelos autores.

Os resultados do teste de equilíbrio de Berg mostraram discreta melhora, com aumento de seis e três pontos para a Criança 1 e Criança 2 , respectivamente, cerca de $13 \%$ e $6 \%$. É possível visualizar a progressão dos parâmetros avaliados na Figura 1.

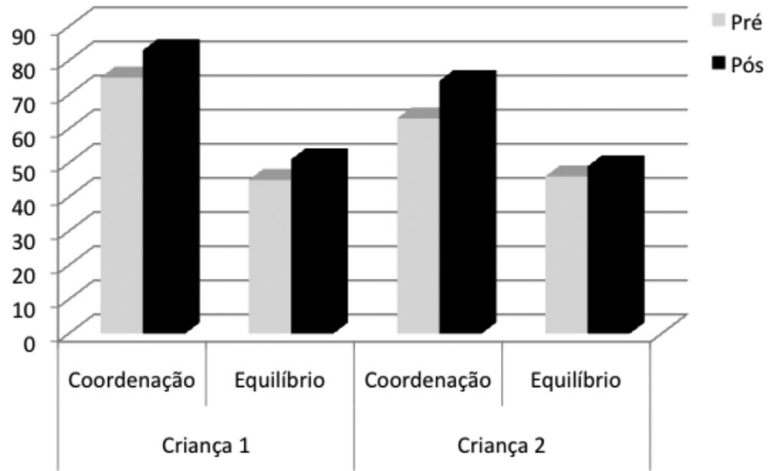

Figura 1: Resultados dos parâmetros avaliados pré e pós treinamento sensório-motor

Fonte: Elaborada pelos autores.

\section{Discussão}

Sabendo-se da influência da deficiência visual no desempenho funcional das crianças e que a propriocepção possibilita adaptações por meio da vivência por atividades e estímulos ${ }^{14,15}$, verificou-se, assim, aperfeiçoamento da resposta motora das crianças avaliadas tanto no teste de equilíbrio quanto no de coordenação motora por progressão nos valores encontrados, sendo que quanto maior a pontuação, melhor a capacidade coordenativa e de equilíbrio.

Na literatura ainda há uma escassez de pesquisas que avaliem as variáveis analisadas para crianças com deficiência visual em fase escolar, por isso os resultados foram discutidos com estudos que possuíam metodologias semelhantes.

Pesquisas correlacionaram déficits nas habilidades sensório-motoras devido à presença de deficiências visuais, como o estudo de Moreno e Paixão ${ }^{4}$ que comparou a avaliação psicomotora entre crianças videntes (grupo controle) e com deficiência visual (grupo de estudo) de ambos os gêneros, também na faixa etária escolar, de 7 a 12 anos, totalizando 44 crianças divididas igualmente entre os grupos. Verificaram que os resultados do grupo estudo foram estatisticamente inferiores ao grupo controle no que diz respeito aos itens da Bateria Psicomotora (BPM), entre eles a praxia global e o equilíbrio. Tal relato se 
confirmou no presente estudo, onde as crianças apresentaram déficit significativo, que foi observado pelos escores e consequente baixa classificação coordenativa obtida na avaliação inicial.

Sá e colaboradores ${ }^{10}$, por meio de avaliação funcional composta por itens sensoriais, motores e visuais, detectaram presença de disfunção sensório-motora em crianças com baixa visão pela privação de estímulos do meio ambiente. Conforme relatado em pesquisas que afirmam que se essas crianças forem estimuladas repetidamente podem, mesmo que lentamente, desenvolver tais domínios de coordenação motora e equilíbrio ${ }^{16,17}$, o presente estudo corrobora com a literatura atual por demonstrar um efeito positivo do treinamento sensório-motor pela evolução da coordenação motora e equilíbrio corporal nas duas crianças com deficiência visual avaliadas, visualizado na avaliação final após a aplicação do protocolo proposto (Figura 1).

Lopes, Kitadai e $\mathrm{Okai}^{13}$ realizaram um trabalho onde aplicaram um programa fisioterapêutico semelhante uma vez por semana durante uma hora, no período de vinte meses, com atividades para trabalho de propriocepção, treino de equilíbrio, marcha e coordenação motora em crianças com deficiência visual. $O$ total de doze crianças foi dividido em dois grupos por faixa etária: um grupo de um a sete anos, e o outro de oito a quinze anos, realizando avaliações para graduar o controle motor e equilíbrio. Os participantes apresentaram falhas principalmente antes do tratamento e obtiveram um ganho de $14 \%$ em média com relação ao equilíbrio após o tratamento e, de forma geral, apresentaram grande melhoria em seu quadro geral e em suas aquisições nos parâmetros avaliados. O que foi possível observar nos resultados do presente trabalho, mostrando que o treinamento realizado contribuiu para a melhora da coordenação e equilíbrio das crianças estudadas.

A Criança 2, que apresenta diagnóstico de cegueira, obteve menor desempenho coordenativo inicial por não possuir memórias construídas por meio de informações visuais e nenhuma aferência visual. Sendo que, após o treinamento, a mesma criança apresentou melhor classificação do desempenho coordenativo. Fatores justificados por Maggi e colaboradores ${ }^{18}$ que realizaram um estudo com 30 crianças, de ambos os sexos com idade entre 10 e 12 anos, sendo 10 com deficiência visual (cegueira congênita total) e 20 com visão normal (com 10 crianças realizando a avaliação de forma vendada) utilizando o Teste de Proficiência Motora de Bruininks-Oseretsky que fornece informações sobre o desempenho da motricidade e equilíbrio. Os resultados obtidos mostraram que o grupo cego congênito teve um desempenho inferior quando comparado com o grupo sem comprometimento visual, sugerindo que a ausência de qualquer tipo de experiência visual interfere na capacidade motora e de equilíbrio.

$\mathrm{Na}$ presente pesquisa verificou-se melhora no equilíbrio, comprovando a influência da estimulação sensorial por outras vias que não a visual para as crianças estudadas, como também sugerem os autores Gallahue, Ozmun e Goodway ${ }^{19}$. Pois, as experiências motoras e proprioceptivas proporcionadas pelo treinamento sensório-motor aplicado objetivaram a orientação corporal e a manutenção do equilíbrio. Os resultados revelaram que a maior evolução com relação ao equilíbrio foi da Criança 1, de 7 anos, por esta ainda possuir alguma visão útil ${ }^{19}$. E as respostas da Criança 2 podem estar relacionadas a cegueira total que leva à lentidão na mobilidade e insegurança de movimentação ${ }^{20}$.

Existe carência de publicações que mostrem a atuação da fisioterapia em crianças com deficiência visual. Apesar da melhora obtida na coordenação e equilíbrio, nenhuma das duas crianças participantes alcançou a classificação coordenativa de coordenação normal ou a máxima pontuação de equilíbrio, sugerindo assim não apenas que ainda há déficit, mas um seguimento em longo prazo, com a aplicação do treinamento em mais atendimentos e intervenções futuras com maior número de indivíduos a fim de um acompanhamento maior. Sendo um fator limitante do presente estudo a dificuldade de obter uma amostra homogênea que atendesse 
aos critérios de inclusão, resultando no tamanho reduzido da amostra.

O programa proposto mostrou-se eficiente para a amostra analisada e contribuiu para a melhora da resposta motora destas crianças (seja baixa visão ou cegueira total), ressaltando a necessidade de enriquecimento e treino proprioceptivo para tais crianças em idade escolar também para ampliar as fontes de informações sensoriais, propiciando mais segurança corporal e consequente melhor coordenação e equilíbrio.

\section{Conclusão}

Foi demonstrado que houve melhora na resposta motora da amostra analisada, pois após os atendimentos foi possível verificar evolução positiva na pontuação obtida em suas respostas de equilíbrio corporal e coordenação motora. Conclui-se que o protocolo utilizado trouxe benefícios para o equilíbrio e resposta motora destas crianças com deficiência visual.

\section{Agradecimentos}

CREFITO 14 (Conselho Regional de Fisioterapia e Terapia Ocupacional) e UESPI (Universidade Estadual do Piauí).

\section{Referências}

1. Silva MB, Shimano SGN, Oliveira CCES, Conti V, Oliveira NML. Avaliação das alterações posturais e retrações musculares na deficiência visual: estudo de caso. Saude Colet. 2011; 49(8): 77-82.

2. Meereis ECW, Lemos LFC, Pranke GI, Alves

RF, Teixeira CS, Mota CB. Deficiência visual: uma revisão focada no equilíbrio postural, desenvolvimento psicomotor e intervenções. R. bras. Ci. e Mov. 2011; 19(1): 108-113.

3. Taira JS, Kawaguchi TS, Bofi TC. A reeducação psicomotora na criança com baixa visão associada à diparesia espástica. Temas desenvolv. 2009; 17(97): 37-41.
4. Moreno MRA, Paixão MCM. Avaliação Psicomotora de Escolares com Deficiência Visual. Rev. neurocienc. 2011; 19(2): 214-220.

5. Navarro AS, Fukujima MM, Fontes SV, Matas, SLA, Prado GF. Balance and motor coordination are not fully developed in 7 years old blind children. ArqNeuropsiquiatr. 2004; 62(3-A): 654-657.

6. Gerzson LR, Pasin JSM. Influência da deficiência visual no desenvolvimento neuropsicomotor. Anais do $3^{\circ}$ Fórum de Fisioterapia da UNIFRA; 29-31 agosto 2012; Santa Maria, Brasil. Santa Maria, Centro Universitário Franciscano; 2012.

7. Sá CG, Bim CR. Análise estabilométrica pré e pósexercícios fisioterapêuticos em crianças deficientes visuais. Fisioter. Mov. 2012; 25(4): 811-819.

8. Soares AV. A contribuição visual para o controle postural. Rev Neurocienc. 2010; 18(3): 370-379.

9. Botelhos DC, Bonfim TR. Influência da informação sensorial adicional no treinamento sensório-motor. Fisioter. Pesq. 2012; 19(3): 268-274.

10. Sá FE, Frota LMPC, Bezerra SC, Almeida AKF, Firmino ALP. Perfil Sensório Motor das Crianças com baixa visão atendidas no setor de estimulação visual do NUTEP. Rev Fisioter S Fun. 2012; 1(2): 29-34.

11. Lima SR, Almeida MA. Iniciação à aprendizagem da natação e a coordenação corporal de uma criança deficiente visual: algumas contribuições. Rev. bras. Cienc. Esporte. 2008; 29(2): 57-58.

12. Carminato RA. Desempenho motor de escolares através da bateria de teste KTK. Dissertação [Mestrado em Educação Física] - Universidade Federal do Paraná; 2010.

13. Lopes MCB, Kitadai SPS, Okai LA. Avaliação e tratamento fisioterapêutico das alterações motoras presentes em crianças deficientes visuais. Rev. bras. oftal. 2004; 63(3): 155-161.

14. Malta J, Endriss D, Rached S, Moura T, Ventura L. Desempenho funcional de crianças com deficiência visual, atendidas no Departamento de Estimulação Visual da Fundação Altino Ventura. Arq Bras Oftalmol. 2006; 69(4): 571-4

15. Willrich A, Azevedo CCF, Fernandes JO. Desenvolvimento motor na infância: influência dos fatores de risco e programas de intervenção. Rev. neurocienc. 2009; 17(1): 51-56.

16. Figueira MMA. Assistência da fisioterapia à criança portadora de cegueira congênita. Rev. Benjamin Constant. 2000; 6(17): 13-33. 
17. Carvalho MP, Santos FM, Nasser RL, Vieira PC, Luckow ELT, Leite AS, et al. Atuação da fisioterapia em deficientes visuais. Hygeia. 2010; 6(10): 20-27.

18. Maggi AB, Souza VR, Sinésio T, Machado DS, Vieira MM. A influência da visão no equilíbrio de crianças deficientes visuais congênitas e com visão normal. Revista Digital EFDeportes.com (Buenos Aires). 2011; Mar.
19. Gallahue DL, Ozmun JC, Goodway JD.

Compreendendo o Desenvolvimento Motor: bebês, crianças, adolescentes e adultos. 7. ed. Porto Alegre: Artmed; 2013. p. 286.

20. Andrade CDA, Gois MLCC, Vitor LGV, Raio JC, Zechim FC, Silva RA et al. Equilíbrio e risco de quedas em crianças com deficiência visual. ConScientiae Saúde. 2012; 11(4): 625-634. 